\title{
THE APPLICATION OF RECOMMENDER SYSTEMS TO DATA-DRIVEN DIGITAL MEMORY
}

\author{
Tingyu Luo and Miguel Baptista Nunes \\ Sun Yat-Sen University \\ Guangzhou, Guang Dong, China
}

\begin{abstract}
Data-driven digital memory applications lack predefined navigation paths and strict hierarchical structures. They are based on large collections of memory items that can become overwhelming to users. Recommender systems can improve user experience through the proposal of personalized relevant items. However, very little academic literature has been dedicated to discussing this type of filtering of digital memory resources and the provision of customized contents to active users. In this paper, an architecture of a hybrid enhanced recommender (HER) system, which integrates collaborative filtering and content based filtering techniques and resolves most of the weaknesses of the individual approaches. This architecture also proposes an ontology to build semantic user profiles and represent memory items to mitigate the lack of semantics of traditional content-based method. Through combining those techniques, this architecture has the potential to cope with data sparsity problems, avoid overspecialization issues and partially resolve cold start problems.
\end{abstract}

\section{KEYWORDS}

Digital Memory, Recommender Systems, Content-Based Filtering, Collaborative Filtering, Ontology

\section{INTRODUCTION}

With the expansion of globalization, the preservation of cultural identity and diversity have become a pivotal issue. The United Nations Educational, Scientific and Cultural Organization (UNESCO) keeps adding cultural heritage sites into the list of World Cultural Heritage. Different countries and local governments are publishing policies and strategies to support cultural preservation projects, whilst organizations such as museums and libraries are encouraged to apply advanced technology to protect and popularize traditional cultures and academic researchers are inspired to do delve in this field. In China and following this trend, the archival academic community has been actively engaging with the concept of "Digital Memory" (Feng, 2012). This type of digital application aims to create internet-based digital resource warehouses based on digital archival resources containing a wide variety of memory media documents in the form of text, pictures, audio, video and others. In China, digital memory applications focus on all aspects of tangible and intangible heritage, history and culture as well as Chinese daily life endeavors, social life and notable events.

This area of research has recently grown both in interest and funding, notably in China, and many publications have emerged in the field of digital humanities focusing on the development of applications that collect and present digital cultural resources online (Luo et al., 2018). This development is partly due to an increasing valuation of cultural identities in a progressive globalized and integrative society and to the increasing number of digital resources emerging on the web and social networks. The key problems in harnessing this increasing amount of diverse and heterogeneous resources are related with organization, namely classification, content generation and presentation. The most common used organization method is classification, such as topical classification, hierarchical classification, classification based on geographic location or event timelines. This resource organization then enables digital memory content generation model as well as user centered presentation designs. Luo and Nunes (2018) identified three ways of generating contents for digital memory applications: data-driven, hierarchical and pre-determined path. Pre-determined content generation model is widely used in story telling projects, in which resources are organized according to pre-defined lenses, aims and navigational paths. This is a "one size fits all" type of application providing fixed paths and fixed layout of resources. Hierarchical models are organized according to pre-defined 
hierarchical classifications. Criteria such as, events, time periods, geographical locations, memorable themes, timelines and specific institutions are often used to aggregate resources. However, this linear classification based on limited feature or events often ignores the value of semantic relationship between different resources and therefore affects the retrieval efficiency. Furthermore, the hierarchy-based content generation model prevents effective customization of the digital resources according to user's needs and preferences and limits user experience. Data-driven content generation model resolves this problem by combining dynamic collection of user's preference data with the semantic characteristics of resource items.

This last generation of digital memory application relies heavily upon recommender systems and provides a dynamic bridge between resources and users. According to Isinkaye et al., (2015), recommender systems are a form of information filtering systems that solve the problem of information overload by filtering vital information fragments out of large amounts of dynamically generated information according to user's preferences, interests, or observed behaviors. This type of systems has been widely used in fields such as e-commerce (Linden et al., 2003), book recommendation (Rana \& Jain, 2012), movie recommendation (Ha et al., 2012), etc. Digital memory data-driven generation models use recommender systems basic theory to enable users to deal with digital memory resources overload. However, there are very different approaches and techniques developed according to different theoretical perspectives, purposes and intended outcomes. Therefore when discussing data-driven digital media there are several potential alternative design architectures that can be considered.

This paper identifies the main-stream recommender filtering techniques and discusses their respective characteristics, features and affordances. The paper then discusses their applicability to digital memory and discusses the relative merits of the different recommendation methods in relation to their capability to deal with digital memory resources as well as user's behaviors and needs. The paper then concludes by proposing a specific architecture for this type of digital application.

\section{LITERATURE REVIEW}

\subsection{Studies on Digital Memory}

Generally speaking, digital memory study involves disciplines such as psychology, sociology, computer science, history, communication, archival science and culture study (Shao, 2014). Memory can be classified into cultural memory, historical memory, individual memory, collective memory, social memory, media memory and digital memory according to the different lenses used by the different disciplinary perspectives. From a thorough analysis of extant literature, the studies addressing digital memory can be classified into 7 aspects:

a. The application of digital records/memory. Researches published on the topic of the application of digital records/memory are mostly about the use of digital records such as social media (Sternfeld, 2011), digital archives (MÜLLER, 2017), online communities in different disciplines' study: history (Milligan, 2012; Chassanoff, 2018), media archaeology (Fyfe, 2016), memory study (Henninger \& Scifleet, 2016), disaster study (Su \& Gibson, 2016);

b. The development of digital memory tools, applications or platforms. Researches about building online digital memory platform involves different topics, some are event-driven, some are people-driven, some focus on interface design, organizing resources or information retrieval, for example: Vicente-Hernández et al (2011), Gulotta et al. (2014), Roiu (2017). In China, digital memory related researches are more focused in this area, including the general discussion of applications' architecture, the technologies which can be applied in terms of protecting culture heritage.

c. The implications of digital memory. Including the implications of the growing impact of information technology, globalization, digitalization on digital memory (Mitra, 2005), (Oiarzabal, 2014). The implications of the increasing number of digital memory platforms and born-digital records on resources curation (Gulotta et al., 2014), humanities (Kunt et al., 2017), history (Slumkoski et al., 2008), policies (Mitchell, n.d.).

d. Strategies, policies, rights and legislation for digital memory. Such as the exploration of strategies implemented to manage electronic resources (Feng \& Zhang, 2010), the protection of privacy (Majovski, 2012), the policies and practices that facilitating corporations (Chigariro, 2014). 
e. Relevant technologies and approaches. Research in this category normally involves the study of specific technology which contributes to the development of digital memory or memory institutions, such as Khan et al. (2009), Seifi (2011), Dobbins et al. (2013) and the study of approaches which improves information management Samiee et al. (2012) and Convery (2011).

f. Factors that affect the development of digital memory, including cultural, political, social and ethical issues Boamah et al (2016) and Glanville (2010).

g. Publications about practical projects and practices.

However, not much literature has been dedicated to discuss data-driven digital memory and the corresponding filtering of digital memory resources through recommender system.

\subsection{Studies on Recommender System}

Recommender systems deal with the problem of information overload by providing users with personalized, exclusive content according to their specific and identifiable interests (Konstan \& Riedl, 2012). Studies on recommender systems can be classified into the study of recommendation filtering techniques, the study on how to evaluate recommender systems and the application of recommender systems in different fields. Recently, different approaches have been developed to build recommender systems commonly divided into collaborative filtering, content-based filtering and hybrid filtering (Isinkaye et al., 2015).

Collaborative filtering is the most mature and widely used technique, it recommends items either by identifying other users with similar rating of interest in different items or by identifying similar items through different user's rating on items. One of the earliest examples of collaborative filtering uses a user-item rating matrix to recommend items to the active user. GroupLens is a classic example of a news recommender system applying memory-based collaborative filtering technique and help users to locate their preferred articles (Resnick et al., 1994). Another common example of collaborative filtering technique is model-based and applies instead machine learning algorithms as proposed by Breese et al. (1998) and Nathanson et al. (2007). Typical successful applications of applied collaborative filtering techniques include the pioneer book recommender systems by Amazon (Ziegler, 2005) and the very early email-based music recommender system Ringo (Chen, 2008).

Content-based filtering techniques are originated from information retrieval theories. The aim of content-based filtering technique is matching items' features to users' preferences, without comparing different users' preferences. One of the basic principles behind this technique is calculating similarity between current item and items which user liked before. Predictions are made based on information of the active user and ignore contributions from other users or items (Celma and Serra, 2008). The core concept behind content-based filtering method is similarity calculation. Music, movies and non-textual items are often filtered through keyword represented contents similarity using Dice Index (Jannach, 2010). Text-based items are often filtered through Cosine Similarity. Other approaches use clustering techniques, such as Billsus et al.(2000), who has applied Nearest Neighbor to find items that match user's short term preference. Manning (2010) has compared the effectiveness of different machine learning information retrieval methods such as SVM, Naive-Bayes, Racchio that are eminently suited to be used in content-based filtering techniques. The use of artificial intelligence (AI) methods in this type of filtering has also been extensively studied. One the earliest pioneers in this area - Pazzani (1999) - proposed an intelligent agent that attempted to predict user web page preference by using a naive Bayesian classifier. With the arrival of the Big Data era, Rao (2012) has presented research on how to apply keyword-based content filtering method in distributed system.

Although collaborative filtering and content-based filtering techniques are widely adopted both in academia and industry, several limitations of these methods have been identified (Adomavicius and Tuzhilin, 2005). Collaborative filtering methods have been found to present problems such as: cold-start; sparsity and scalability. On the other hand, content analysis methods of the present problems of overspecialization and cold-start.

As a result, hybrid filtering which combines two or more filtering techniques are proposed to improve the quality of recommendation. The rationale of the combination is to maximize the merits and minimize the weaknesses of the individual recommender method. Hybrid algorithms commonly use both items' attributes and the ratings of users, some also incorporate additional external information and knowledge sources into the recommender processes. Jannach et al. (2010) have categorized hybrid strategies into: integrated mixing, 
parallel mixing and linear mixing. Integrated mixing such as hybrid feature combination often integrates data used in different techniques and then apply this data into selected filtering technique. For instance, Basu (1998) has combined a form of collaborative preference feature with content catalog to determine peers in the same community. Parallel mixing, often runs different recommender techniques at same time and adopt a strategy to combine the result from those subsystems. A common example of parallel mixing is weighted hybrid, where the prediction score results from the calculation of the weighted average of predicted values from different recommendation techniques. Linear mixing approaches, such as switching and cascade hybrid often employ different recommender techniques sequentially. Tran (2000) presents an example where he developed an architecture of a hybrid recommender system which can switch filtering techniques according to user's behavior and rating data.

Although this paper focus on the recommender system architecture, it should be acknowledged that research on recommender systems also addresses many other aspects, such as design of the interface; human-computer interactions; holistic evaluation of the system.

\section{CRITICAL ANALYSIS ON RECOMMENDATION FILTERING TECHNIQUES FOR DIGITAL MEMORY}

As stated above, this paper aims to discuss the applicability of recommender systems to digital memory and discuss the relative merits of the different recommendation methods in relation to their capability to deal with digital memory resources and user's needs. Since, recommendation filtering technique are the core component of recommender systems this critical analysis evaluates the three main types of recommender systems filtering techniques identified above against their potential in the specific context of digital memory. Furthermore, as discussed in the introduction, this paper specifically focusses on data-driven digital memory applications that most benefit for recommender systems. In data-driven digital memory the repository of memory items (data) can be (driven) retrieved, analyzed, manipulated and represented according to user's expectations, culture, and social anticipations as well as personal perspectives (Luo and Nunes, 2018). The recommender systems can therefore be the drivers of the memory representation being dynamically offered to the active user.

\subsection{Collaborative Filtering and Digital Memory}

According to Halbwachs (1992), the continuous necessity for extracting and preserving collective memory for social use and keep group consciousness and identity is the main reason for the need for digital memory applications. The intention is therefore that this collective memory can be revisited and experienced by current generations and left as an inheritance for future ones. A person may have agency in several social networks each of these exhibiting a specific group identity, thus recognizing users characteristics is pivotal in recommending memory resources. Collaborative filtering is a domain independent technique which predicts preferences of an active user based on other users' similar interests and choices. This makes it imminently suitable to be used in digital memory applications. Furthermore, collaborative filtering techniques do not give recommendations directly base on explicit statements of user's social network members, but people who give same ratings on different contents potentially indicate their similar identity. This is extremely useful in eliminating any bias or prejudiced profiling.

For instance, an item matrix can be developed to store user's rating on each item - a user-item matrix - and then Pearson correlation or cosine correlation can be used to calculate similarity between different users. Based on this similarity, a cluster of candidate of users can be selected that is estimated to be similar to the current active user and then used to predict which items the current user may be interested in. Alternatively, collaborative filtering methods can also use model-based techniques to develop a classifier or cluster to predict weather a given user will like a certain item or not. The model can be constructed using machine learning and data mining techniques such as decision trees, clustering algorithms, neural network and regression analysis. After the model is built, the system can then automatically make predictions based on user's rating and give recommendations.

However, there are some limitations of collaborative filtering techniques as mentioned in literature review. One of the most significant problems is cold-start, which characterizes the phenomena emerging from the lack of data necessary for similarity calculations related to new users that just started to browse the system or new 
items that have just been added. As for digital memory applications, it is impossible to have all required data in the system on users and items, when the system is initially built. Furthermore, in data-driven digital media new users and items will be continuously added, so when applying collaborative filtering techniques the system must routinely solve cold-start problems both for new users and new items. Another problem with collaborative filtering in data-driven digital media is data sparsity. For a digital memory application which may have thousands items, it is unlikely that users are able to browse through all of those items one by one and give ratings on each of them. Normally users will only rate a small set, so the user-item matrix may become a sparse matrix. If the data is too sparse, it will affect the quality of recommendation as it is hard to find neighbors.

In conclusion, although collaborative filtering technique is good in recommending digital memory resources, due to the nature of collective memory building and the need to for the rating of items, problems of cold-start and sparsity may hinder the efficiency and efficacy of the recommending process.

\subsection{Content-based Filtering and Digital Memory}

Content-based filtering technique is a domain related method in which the recommendation is heavily reliant on the characterization of items, as for text-based contents, features can be computed automatically so it is widely used in recommending news, web pages and publications. In digital memory applications using content-based recommender system, items and user profiles are both represented through feature-based vectors. For text-based items, features are automatically selected through TF-IDF - an acronym for "term frequencyinverse document frequency", which is a statistic that is intended to reflect how important a feature is to an item in a dataset (Jannach et al., 2010). For none text-based items, such as videos and images, resources can be annotated by metadata that describe accurately their main attributes. Once the feature-based vectors is computed for all items, similarity between user profile and items is then calculated through cosine correlation.

As content-based filtering techniques also rely on user's past data, it cannot avoid the problem of user cold-start. However, it can solve item cold-start problem and the data sparsity problem through exploiting content information. In addition, this filtering technique is more sensitive to the changes of user's preference, it can give timely recommendation based on user's current preference. The disadvantage of content-based filtering technique is that the recommendation is heavily reliant on descriptions of items. However, in order to better manage, preserve and retrieve digital memory resources, every item has to be coded according to predefined meta-data and stored in XML or RDF format. So, what is perceived as a disadvantage in other contexts, proves to be a benefit when applying content-based filtering techniques to recommend digital memory resources. However, this type of filtering approach may result in overspecialization and lack of serendipity. Overspecialization refers to the problem that users are restricted to getting recommendations similar to items already defined in their profiles (Zhang and Vijay, 2002). Lack of serendipity is closely related to overspecialization, it could even be seen as a consequence this problem, in the sense that it prevents the finding of unexpected or unintended items that could have been found through browsing for instance (Foster and Ford, 2003). Another shortage of content-based techniques is the concentration on simplistic user recommendations and meta-data ignores quality aspects on the item. For example, a digital memory item maybe recommended to a user just because that same user liked items in the same topic as before. In this case, there is not quality control over the items being proposed; only similarity measures.

Knowing what affect user's needs on particular domain is pivotal to content-based filtering technique since we need to decide what features should be included to describe available items. As a result, to implement content-based filtering techniques, the first step is to understand user's information needs related to particular digital memory resources, and then organize items according to these needs. In data-driven digital memory recommender systems, both user profiles and memory contents are represented using the same feature vector space. After that, similarity between users' profile and each item can be calculated and contents, which do not match with user's interest, would be filtered out by setting an appropriate threshold.

In conclusion, due to the rich meta-data description of digital memory resources, content-based filtering techniques can be used in recommending digital memory resources, and this technique successfully avoids the disadvantages of collaborative filtering techniques, such as data sparsity and item cold-start problems. However, due to overspecialization and considering only the preferences of one individual user, it is hard to give recommendations that enable serendipity discovery and good quality assurance. 


\subsection{Hybrid Filtering Techniques and Digital Memory}

As mentioned before, hybrid filtering techniques are developed to try to resolve limitations of applying single filtering methods. Table 1 contrasts the collaborative filtering approaches and content-based approaches, identifying the advantages and disadvantages of each in digital memory resources recommendation. The third row suggests what might be achieved in an ideal hybrid which combines the two techniques. In more recent years, increasing number of researchers are exploring how to incorporate additional side information and external knowledge resources into the content-based recommendation process to improve its shortages (Lops et al., 2019.). One of the widely adopted approach to access external knowledge is linked open data as proposed by (Di et al., 2012) and (Musto et al., 2017). Melville et al. (2002) also discussed how to improve data sparsity problem of collaborative filtering through content-boosted method.

As the result of the previous critical analysis and discussion the final section of this paper proposes an enhanced integrated mixing hybrid recommender system that maximizes the combined effects of the individual techniques in digital memory. This architecture is enhanced because it includes a pre-profiling component to resolve the cold-start problem and also adopts a domain ontology to solve the overspecialization problem of content-based filtering technique.

Table 1. Comparison between collaborative filtering techniques and content-based techniques

\begin{tabular}{|c|c|c|}
\hline Technique & Advantages & Disadvantages \\
\hline Collaborative Filtering & $\begin{array}{l}\text { A. Using the concept of social group to give } \\
\text { recommendation which is essential to social } \\
\text { memory. } \\
\text { B. Personalized recommendation. }\end{array}$ & $\begin{array}{l}\text { E. Data sparsity. } \\
\text { F. Cold-start (user and item). }\end{array}$ \\
\hline Content-based Filtering & $\begin{array}{l}\text { C. Sensitive to short-term variance. } \\
\text { D. No ramp-up required. }\end{array}$ & $\begin{array}{l}\text { F. Cold-start (user) } \\
\text { G. Overspecialization. }\end{array}$ \\
\hline Ideal Hybrid & $\begin{array}{l}\text { Maintains: A, B,C,D } \\
\text { Resolves: E, F(item cold start resolves) }\end{array}$ & F(user cold start not resolved) \\
\hline External Knowledge & $\begin{array}{l}\text { Maintains: A, B,C,D } \\
\text { Resolves: E and G }\end{array}$ & F(user cold start not resolved) \\
\hline
\end{tabular}

\section{A THEORETICAL PROPOSAL OF AN ARCHITECTURE FOR AN ONTOLOGY-BASED HYBRID ENHANCED RECOMMENDER SYSTEM FOR DIGITAL MEMORY APPLICATIONS}

Figure 1 shows this research's proposal for an architecture of a Hybrid Enhanced Recommender (HER) system. This architecture consists of 7 components: the Interactive Interface Agent (IIA), the Pre-Profiling module, the Collaborative Filtering Engine (CFE), the Content-based Engine (CBE), Database of User's Rating, Digital Memory Items Database.

The main job of IIA is to interact with users in different situations such as guiding users through registration and login, getting user's feedback for each recommended item, view and change of user's interest profiles.

As discussed in section 3.2, the item cold-start problem is successfully resolved through applying content-based method, but the user cold-start problem still remain. This architecture proposes a pre-profiling module to deal with this problem. If a new user registers, the interface will guide the user to rate a group of representative items and few other random items, these ratings are then passed to database of user's rating and are used to learn their profile. 
The goal of CFE is to find similar user of the active user and extract terms of their preferred contents and pass this result to CBE. It first receives user's login information from the IIA and query the database of user's rating to get the ratings on items of that user, the ratings on items of other users. Then, the CFE finds the neighbors of the active user through calculating the similarity scores between that user and other users. User profile is then represented by top $\mathrm{N}$ features which reflect the preference of its neighbors and are extracted from the items which they liked before.

The purpose of CBE is to recommend items that best represent the group's preference to which the active user is assigned to. To mitigate the lack of semantics and overspecialization issues of the traditional content-based method, a subject ontology was adopted to represent and integrate the features of digital memory items and user profiles. The idea is to use a semantic network and a spreading activation strategy to infer features of items the user may be interested in. The CBE then matches the semantic user profile against semantic item representations and produces a ranked list of potentially interesting items. The list will then be presented to the user though the IIA, so that the active user can view and rate items. The CBE also modifies the user's interest profile after receive user's rating on recommended items.

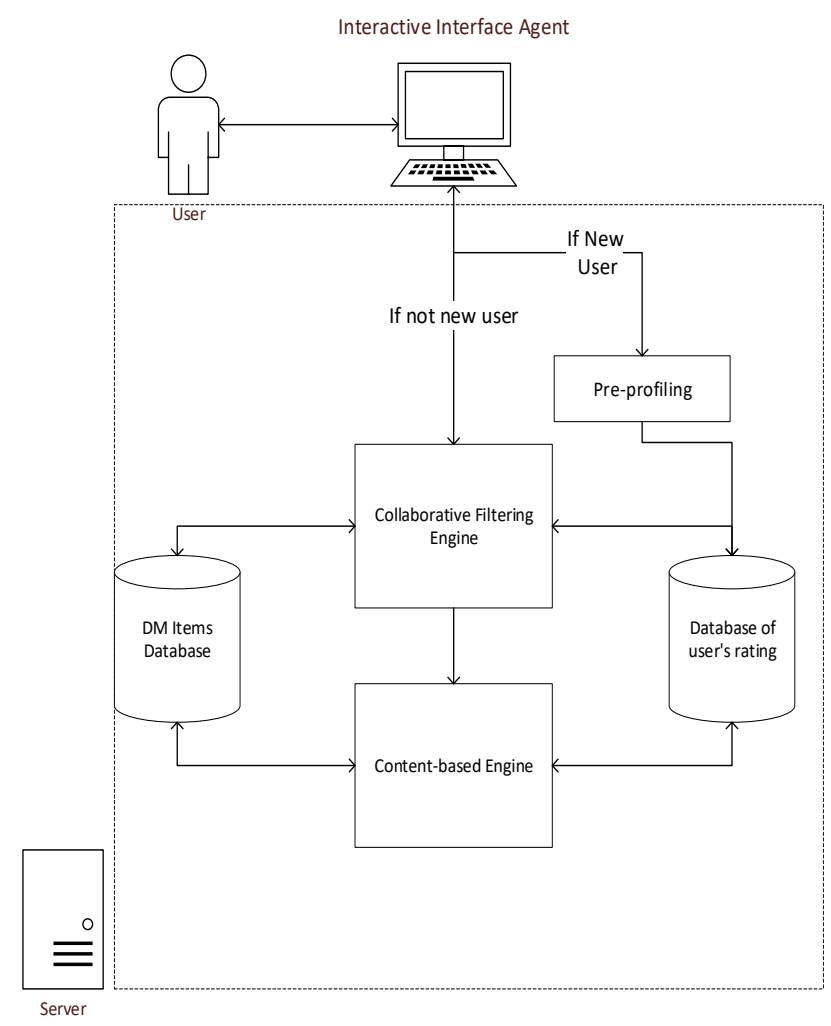

Figure 1. Architecture for integrating Collaborative filtering and Content-based filtering techniques

\section{CONCLUSION}

Based on a critical analysis of different information filtering techniques against the nature of digital memory, this paper proposes an architecture for a hybrid enhanced recommender (HER) system. It is expected that this HER architecture will increase satisfaction and boost user experience of data-driven digital memory applications by providing digital memory resources based on user's preference data. This architecture integrates collaborative filtering and content-based filtering techniques in order to resolve individual weaknesses of these filtering techniques. In addition, it proposes the application of subject ontologies to build 
up semantic user profile and item representations. Such a hybrid recommender system will have all the advantages of both techniques, namely applying the concept of social group to give recommendations which are essential in socially constructed memory; giving personalized recommendations; exhibit sensitivity to short-term variance; and no ramp-up issues. Additionally, it uses a pre-profiling component to avoid a problem common to both individual techniques: user cold start problem.

\section{REFERENCES}

Adomavicius, G., \& Tuzhilin, A. 2005. Personalization technologies: A process oriented perspective. Communications of the ACM, Vol.48. No.10, pp. 83-90.

Basu, C., et al. 1998. Recommendation as classification: Using social and content-based information in recommendation. AAAI Technical Report WS-98-08, pp. 714-720.

Billsus D, et al. 2000. A learning agent for wireless news access. In Proceedings of the 5th International Conference on Intelligent User Interfaces, New Orleans, USA, pp. 33-36.

Boamah, E. et al. 2016. Cultural, social and political factors influencing the adoption of Digital Preservation Management in Ghana. Innovation: Journal of Appropriate Librarianship and Information Work in Southern Africa, Vol.53, pp. 6-20.

Breese, J. S. et al. 1998. Empirical analysis of predictive algorithms for collaborative filtering. In Proceedings of the Fourteenth conference on Uncertainty in artificial intelligence. Madison, USA, pp. 43-52.

Celma, Ò., \& Serra, X. 2008. FOAFing the music: Bridging the semantic gap in music recommendation. Web Semantics: Science, Services and Agents on the World Wide Web, Vol.6, No.4, pp. 250-256.

Chen, L. S. et al. 2008. Developing recommender systems with the consideration of product profitability for sellers. Information Sciences, Vol.178, No.4, pp.1032-1048.

Chassanoff, A. M. 2018. Historians' Experiences Using Digitized Archival Photographs as Evidence. The American Archivist, Vol.81, No.1, pp. 135-164.

Chigariro, D. 2014. Collaboration in the management and preservation of audio-visual archives: a case study of the National Archives of Zimbabwe. Doctoral dissertation, University of Western Cape.

Convery, N. 2011. Information management, records management, knowledge management: The place of archives in a digital age. In The future of archives and recordkeeping: A reader. pp. 190-212.

Di Noia, T et al. 2012. Linked open data to support content-based recommender systems. In Proceedings of the 8th International Conference on Semantic Systems. New York, USA, pp. 1-8.

Dobbins, C. et al., 2013. Exploiting linked data to create rich human digital memories. Computer Communications, Vol. 36. No.15-16, pp. 1639-1656.

Foster, A and Ford, N. 2003 Serendipity and information seeking: an empirical study. Journal of Documentation, Vol. 59 Issue: 3, pp. 321-340

Isinkaye, F., Folajimi, Y., \& Ojokoh, B. 2015. Recommendation systems: Principles, methods and evaluation. Egyptian Informatics Journal, Vol.16, No.3, pp. 261-273.

Müller, K. 2017. Between Lived and Archived Memory: How Digital Archives Can Tell History. Retrieved June 17, 2018, from http://www.redalyc.org/html/550/55051207002/

Nathanson, T. et al. 2007. Eigentaste 5.0: constant-time adaptability in a recommender system using item clustering. In Proceedings of the 2007 ACM conference on Recommender Systems, Minneapolis, USA, pp.149-152.

Konstan, J. A., \& Riedl, J. 2012. Recommender systems: from algorithms to user experience. User Modeling and User-Adapted Interaction, Vol. 22, No.1-2, pp.101-123.

Oiarzabal, P. J. 2014. Migrant Memories in the Ephemeral Digital Age: The Case of the Basque Institutional Diaspora in North America. Identity Palimpsests: Archiving Ethnicity in the US and Canada, Vol. 297, No.314, pp. $297-314$.

Pazzani, M. J. 1999. A framework for collaborative, content-based and demographic filtering. Artificial Intelligence Review, Vol.13, pp.393-408.

Feng, H. L, \& Zhang, N. (2010). Developing a National Strategy for Electronic Records Management (ERM) for China: Key Features IQ: The RIM Quarterly. Vol.26, No.1, pp. 35-37.

Feng, H. L. 2012. 档案记忆观、资源观与"中国记忆"数字资源建设. 档案学通讯, Vol.3, pp 4-8.

Fyfe, P. 2016. An Archaeology of Victorian Newspapers. Victorian Periodicals Review, Vol.49, No.4, pp. 546-577.

Glanville, L. (2010). Web archiving: Ethical and legal issues affecting programmes in Australia and the Netherlands. Australian Library Journal, Vol.59, N0.3. pp. 128-134. 
Gulotta, R. et al. 2014. Legacy in the Age of the Internet: Reflections on How Interactive Systems Shape How We Are Remembered. In Proceedings of the 2014 Conference on Designing Interactive Systems. Vancouver, Canada. pp. 975-984.

Ha, I., Oh, K. J., Hong, M. D., \& Jo, G. S. 2012, November. Social filtering using social relationship for movie recommendation. In Proceedings of the International Conference on Computational Collective Intelligence. Berlin, Germany. pp. 395-404.

Halbwachs, M. 1992. On collective memory. University of Chicago Press.

Henninger, M., \& Scifleet, P. (2016). How are the new documents of social networks shaping our cultural memory. Journal of Documentation, Vol.72, No.2, pp. 277-298.

Jannach D. et al. 2010. Recommender systems: an introduction. Cambridge University Press. Cambridge, England.

Khan, H., et al., 2009. The International Journal of Digital Curation, Vol.4, No.3. pp. 209-221.

Kunt, G., Székely, J., \& Vajda, J. (2017). Making (DIS)Connections: An Interplay Between Material and Virtual Memories of The Holocaust in Budapest. Retrieved from http://www.lidemesta.cz/assets/media/files/19-2017-2/Kunt.pdf

Linden, G., Smith, B., \& York, J. 2003. Amazon.com recommendations: Item-to-item collaborative filtering. IEEE Internet computing. Vol.1, pp76-80.

Lops, $\mathrm{P}$ et al. 2019. Trends in content-based recommendation. User Modeling and User-Adapted Interaction, Vol.29. No.2. pp.239-249.

Luo, T. Y. et al., 2018. Establishing a digital memory application model from an information science perspective: A systematic literature review. In Proceedings of the 11th International Conference on ICT, Society, and Human Beings 2018, Madrid, Spain.

Luo, T.Y., \& Nunes, M. 2018. Conceptual Integration of Archives, Data, Information and Information Technology for Digital Memory Applications: The CMDMA Model. In Proceedings of 2018 the European Association for Digital Humanities Conference. Galway, Ireland.

Majovski, K. 2012. Data Expiration, Let the User Decide: Proposed Legislation for Online User- Generated Content. University of San Francisco Law Review, Vol. 47, pp. 807-838.

Manning, C. et al. 2010. Introduction to information retrieval. Cambridge University Press. Cambridge, England.

Melville, et al. 2002. Content-boosted collaborative filtering for improved recommendations. In Proceedings of the 20th Conference on Innovative Applications of Artificial Intelligence, Vol.23, pp.187-192.

Milligan, I. 2012. Mining the 'Internet Graveyard': Rethinking the Historians' Toolkit. Journal of the Canadian Historical Association, Vol. 23, No.2. pp. 21

Mitchell, C. L. Informationalism and Katrina, Analyzing How Digitalization Affects Policy Making. Retrieved from http://citeseerx.ist.psu.edu/viewdoc/download?doi=10.1.1.539.912\&rep=rep1\&type=pdf

Mitra, A. 2005. Digital Memory. Journal of Information, Communication and Ethics in Society Kybernetes, Vol.12, pp. 3-13.

Musto, C. et al. 2017. Semantics-aware Recommender Systems exploiting Linked Open Data and graph-based features. Knowledge-Based Systems, Vol.136, pp.1-14.

Rao W et al. 2012. Move: A large scale keyword-based content filtering and dissemination system. IEEE 32nd International Conference on Distributed Computing Systems; Macau, China. pp. 445-454.

Rana, C., \& Jain, S. K. 2012. Building a Book Recommender system using time based content filtering. WSEAS Transactions on Computers, Vol.11, No.2, pp 2224-2872.

Resnick, P. et al. 1994. GroupLens: an open architecture for collaborative filtering of netnews. Proceedings of the 1994 ACM conference on Computer supported cooperative work. Chapel Hill, USA, pp. 175-186.

Roiu, C. I. 2017. The Challenge of Multiple Identities - Multi-ethnicity in Genealogy, Local History and Regional Memory - Challenges and Opportunities for Libraries and Other Memory Institutions. In Proceedings of the IFLA 2017 Satellite Meeting on Genealogy and Local History, Martynas Mažvydas National Library of Lithuania, Vilnius, Lithuania, pp. $1-10$.

Samiee, M., et al. 2012. Proposing a paradigm for the management of digital images of the NLAI(National Archive of Iran) according to the Open Archival Information System(OAIS) Reference Model. In Proceeding of the Archiving Conference, Archiving 2012 Final Program and Proceedings. Vol. 2012, No. 1. pp. 153-158.

Seifi, L. 2011. Digitization and digital preservation of manuscripts and access in organization of Libraries, Museums and Documents Centre of Astan Quds Razavi Library in IRAN: A case study.

Shao, P. 2014. 媒介作为人类记忆的研究-以媒介记忆理论为视角. Doctoral dissertation, 浙江大学.

Slumkoski, C., et al., 2008. History on the Internet: The Atlantic Canada Portal. Journal of History on the Atlantic Region, Vol. 48, No.1, pp. 132-55.

Sternfeld, J. 2011. Archival Theory and Digital Historiography: Selection, Search, and Metadata as Archival Processes for Assessing Historical Contextualization. The American Archivist, Vol.74, pp 544-575. 
Su, C., \& Gibson, P. L. 2016. Digitizing the Memorial: Institutional and Vernacular Remembrances of the Taiwanese 921 Earthquake and Typhoon Morakot. In A. Hajek, C. Lohmeier, \& C. Pentzold (Eds.), Memory in a Mediated World. Palgrave Macmillan. pp. 74-89.

Tran, T., \& Cohen, R. 2000. Hybrid recommender systems for electronic commerce. In Proceedings of the Workshop on Knowledge-Based Electronic Markets, AAAI Press. University of Waterloo, Ontario, Canada Vol. 40. pp. 78-84

Vicente-Hernández, L. et al., 2011. The Almirall Project: a portal of 19th century culture and thinking. In Proceeding of the IFLA Satellite Conference: A Bridge to Knowledge for Sustainable Development. La Havana, Cuba.

Zhang, T. and Vijay, S. 2002 Recommender systems using linear classifiers. Journal of Machine Learning Research; Vol. 2, pp. $313-34$

Ziegler, C. N., et al. 2005. Improving recommendation lists through topic diversification. In Proceedings of the 14th International Conference on World Wide Web. Seoul, Korea. pp. 22-32. 